\title{
Specimen Preparation Method for Size Distribution Measurements of Nano- materials by Scanning Electron Microscopy - Fixing of Nano-particles on a Substrate with Adhesive Coating
}

\author{
Kazuhiro KUMAGAI $^{1}$ and Akira KUROKAWA ${ }^{1}$ \\ 1. Surface and Nano-Analysis Section, Nanomaterial Characterization Division, National Metrology \\ Institute of Japan, National Institute of Advanced Industrial Science and Technology (AIST), Tsukuba \\ JAPAN
}

In response to the regulations on manufactured nano-materials (NMs), which started in several EU countries based on the definition of NM by European Commission [1], there are increasing discussions to find the solution for the size distribution measurements of NMs to test if a product meets the definition or not [2]. As one of approaches to the regulation, electron microscopies (EMs) such as transmission electron microscopy and scanning electron microscopy (SEM) attract attentions as NM measuring tools, due to their capability to easily and quickly observe individual nano-particles (NPs) [3].

For size distribution measurements by EMs, specimen preparation is one of the most important processes. To achieve reliable measurements, the particles in a micrograph should be clearly visualized and easy to analyze. To minimize ambiguity, particles should be (1) uniformly placed over a specimen holder with no localization in particle size and number, or (2) placed only in a small area that allow us to count all particles in the area. For both of these cases, particles are expected not to overlap each other. Furthermore, it would be helpful, if there are neither agglomerating nor aggregating particles. It is, however, not easy to make such specimens, because NMs, which are usually supplied as suspension, often get localized and aggregated in drying process.

In this paper, we present a preparative method by using polycationic adhesive coating on a substrate to make specimens that satisfy the above condition (1). The approach with adhesive coating has been used in the research field of biology to fix samples such as cell onto a substrate for SEM observations [4,5]. In this study, we applied this technique to important and basic NMs: Au, silica and polystyrene NPs. To form a polycationic adhesive layer, mirror polished Si substrates were dipped in $0.01 \%$ Poly-L- lysine (PLL) solution (Trevigen Inc.) for 2 hours, then rinsed in ultrapure water. These PLL coated Si substrates were dipped into NP suspension to adsorb NPs for 10, 30, 60 and 90 minutes, and then rinsed in ultrapure water. Specimens without PLL coating were also prepared in the same manner. For SEM image observation, we employed a SEM equipped with a filed-emission gun (JSM-7100F, Jeol Ltd.).

Figures 1a-1d show secondary electron (SE) images of Au NPs (30 nm in diameter) fixed on PLL coated Si substrate at four different dipping times. These SE images show that Au particles are randomly and individually fixed on the substrate with no overlapping or agglomeration. On the other hand, the Si substrate without PLL layer hardly adsorbs Au NPs even after 90 minutes dipping (Fig. 1e) that corroborates the effect of PLL layer. The variation of coverage of Au NPs against dipping time is plotted in Fig. 1f. The coverage, namely number of particles, linearly increases with dipping time, which can realize easy tuning of particle density on the substrate. This method is applicable to not only Au NPs, but also silica NPs and polystyrene NPs as shown in Figure 2.

We demonstrated that PLL coating useful to fix NPs on Si substrate uniformly and individually. Our 
method could be effective to a wide range of NMs that are negatively charged in suspension, and offers easy specimen preparation for size distribution measurement of NMs by SEM.

\section{References:}

[1] European Commission, Official Journal of the European Union L 275, 20.10.2011 (2011) p. 38.

[2] A Lopez-Serrano et al, Analytical Methods 6 (2014) p. 38.

[3] EFSA Scientific Committee, EFSA Journal 9 (2011) p. 2140.

[4] SK Sanders et al, The Journal of Cell Biology 67 (1975) p. 476.

[5] K Tsutsui et al, J. Electron Microsc. 25 (1976) p. 163.

[6] The authors thank Dr. H. Kato in AIST for kind help in preparation of the silica NPs. This work was performed as one of the studies conducted by the consortium for measurement solutions for industrial use of nanomaterials (COMS-NANO) in Japan.
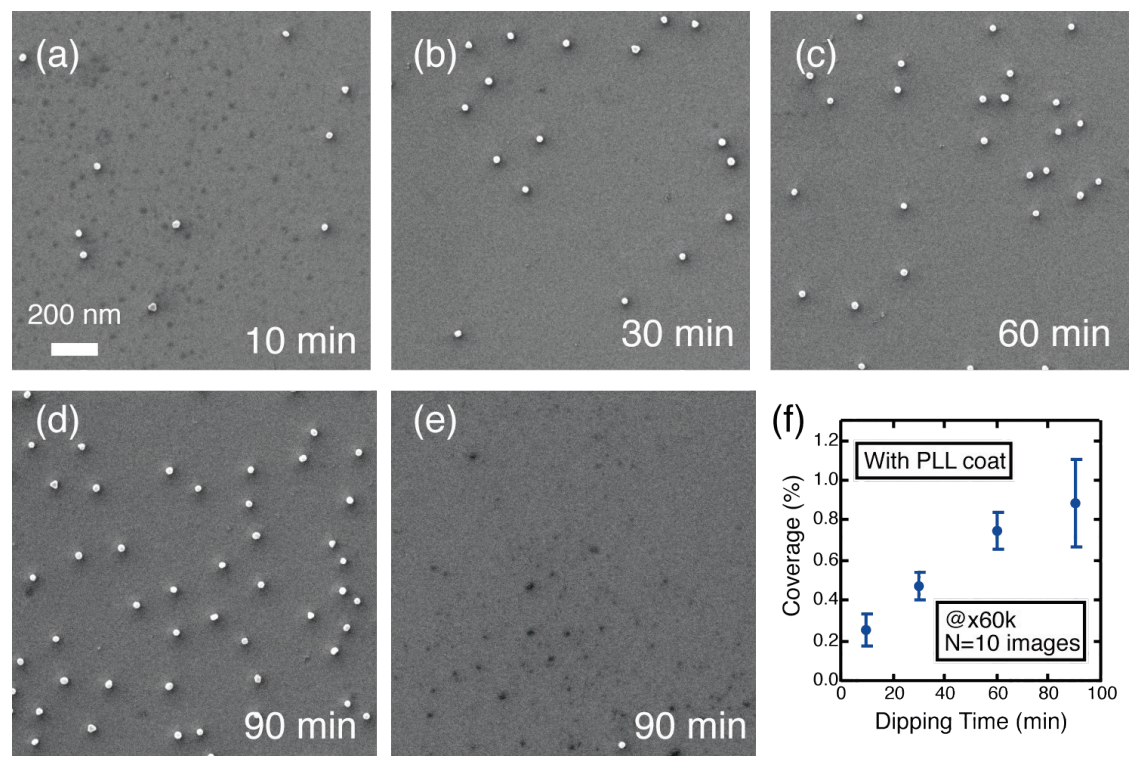

Figure 1. (a)-(d) SE images of Au NPs on PLL coated Si substrate. The dipping time was 10, 30, 60 and 90 minutes, respectively. (e) The same as (d) but without PLL coating. Primary beam energy was $30 \mathrm{keV}$ for all SE images. (f) A plot of coverage of Au NPs against dipping time.
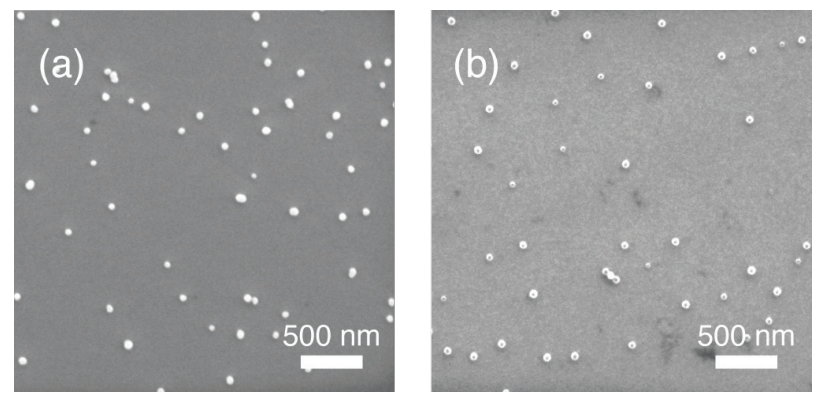

Figure 2. SE images of NPs on PLL coated Si substrate. (a) Silica NPs. Primary beam energy was 3 $\mathrm{keV}$. (b) Polystyrene NPs. Primary beam energy was $4 \mathrm{keV}$. The average particle diameter of both of the materials is $50 \mathrm{~nm}$. 